\title{
The Effects of Charge Persistence in Aladdin III InSb Detectors on Scientific Observations
}

\author{
Randall D. Campbell ${ }^{1}$ and David J. Thompson ${ }^{2}$ \\ 1) W.M. Keck Observatory 2) California Institute of Technology
}

\begin{abstract}
Charge persistence is signal that remains on a detector array after the illumination source has been removed. It is a characteristic of many types of photo detectors but is particularly prominent in near-infrared arrays that utilize Indium Antimonide (InSb) as a photosensitive material. Persistence can be a significant issue for a scientific observation with the potential of reducing the quality of the data. We have studied the properties of persistence for an Aladdin III array from Raytheon which is installed in the Keck Observatory's adaptive optics camera, NIRC2. In this paper we discuss the types of observations that are at risk from persistence effects. We present data on the characteristics which include the following results: 1) the level of persistence is more strongly influenced by incident flux than that of fluence (flux x integration time), 2) the persistence is wavelength independent, 3) the persistence level is not reduced by continuous reset-reads of the array or "flushing", 4) the determination that the persistence decay scales with a power law and is proportional to $1 /$.
\end{abstract}

Keywords: $\quad$ Persistence, Charge trapping, Image Latency, Indium Antimonide, InSb, Aladdin

\section{Introduction}

Charge persistence of photo-detectors, sometimes referred to as latency or memory, is defined as the signal that persists on the array after the illumination source has been removed from the field of view, Solomon et al. (1993). The residual charge decays with time and can result in significant amounts of unwanted signal in subsequent images. Many types of detectors can have this characteristic but near infrared detectors with indium antimonide, $\mathrm{InSb}$, as the photo-sensitive material are particularly susceptible to this phenomenon. Solomon (1999) concluded that the cause of persistence is impurity sites in the passivation layer that can trap holes which then tunnel back into the InSb layer. In this paper we explore the characteristics of charge persistence, some of its effects on ground based astronomy, and strategies to minimize the difficulties persistence can present to astronomers.

\section{Difficulties of Persistence}

NIRC2 is an imager and spectrometer for Keck II's adaptive optics system that uses an Aladin-3 array, a 1024x1024 InSb developed by Ratheyon Vision Systems, RVS, Fowler et al. (1996) . NIRC2 is both an imager and a spectrometer and since there is no slit viewing camera the images of the science target must be used to align the spectrometer slit. The difficulties of persistence in NIRC2 can be significant because the higher fluxes from direct imaging create persistence effects that are a problem for the subsequent spectroscopic integrations. Since persistence is a current source that gradually leaks out, the longer spectroscopic integrations are particularly susceptible to this effect. The problem is illustrated in figure 1, which is a 1200 second exposure of a 
crowded field near the galactic center. The image in figure 1 contains many features, including horizontal continuum spectra from several stellar sources, emission features from hot gases, residual absorption and emission telluric features of from materials in the earth's atmosphere, and artifacts from bad pixels, hot pixels, and cosmic rays. However, the source of the largest signal in this 1200 second integration is charge persistence from the previous image, in spite of the fact that care was taken to protect the array from bright sources which could create the trapped charge. The array region of interest where the spectra were scientifically interesting was protected as the observers went to extraordinary lengths to keep the array quiet prior to obtaining the spectra. This included observing blank-sky spectra prior to the science field, shuttering the camera when slewing, pre-imaging at very short exposure times with a narrow band filter to not saturate, and using nod patterns that moved the spectra of interest away from regions that are contaminated with persistence. Even with these precautions one can see that the data quality could easily have been degraded if a careful observing plan was not followed. Persistence is a major problem for imaging spectrographs (like NIRC2), when using the instrument as an imager prior to spectroscopic observations. However, observing bright sources like standard stars or the high flux background in the thermal infrared will still cause problems for subsequent faint-object spectroscopy in pure spectrographs.

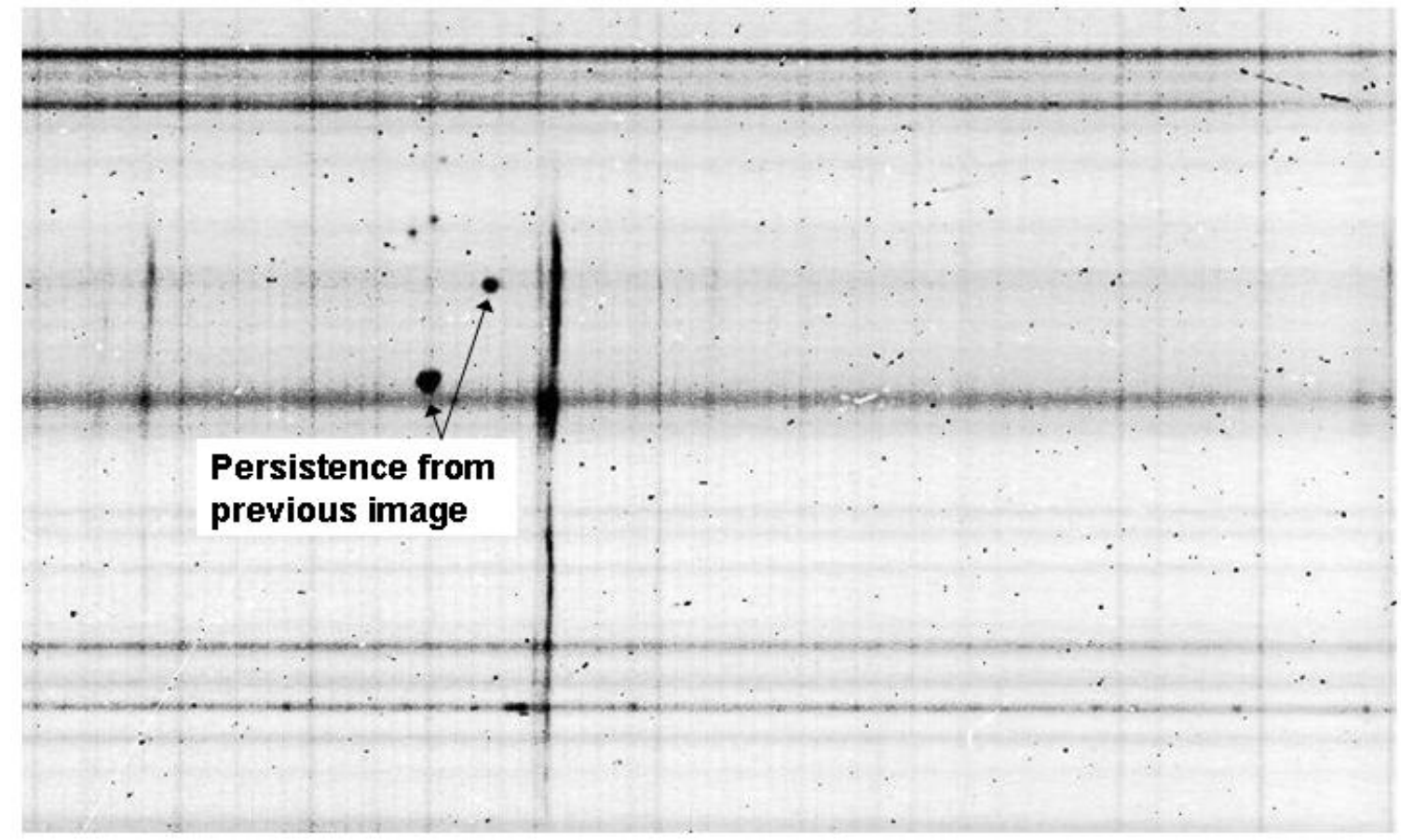

Figure 1: Persistence Contamination Example: NIRC2 K-band (2.0-2.5 $\mu \mathrm{m})$ long-slit spectra from a crowded region near the Galactic Center (photo credit A. M. Ghez).

The image includes continuum spectra from several point sources and emission features from hot ionized gases. There are persistence features from a previous direct image evident in the array. These features have a strong signal and can easily contaminate the data if not carefully accounted for. These data were acquired with a1200 second integration. Since charge persistence is effectively a current source, longer integrations used for acquiring spectra are very susceptible to persistence effects. 


\section{Characterization of Persistence}

In order to help avoid the troubles associated with persistence, we collected experimental data in an effort to better understand the character of this phenomenon. In the case of NIRC2, persistence was analyzed while the instrument was being developed in the Caltech laboratories and additional measurements were conducted after the instrument was delivered to the Keck II telescope.

Several experiments were setup to quantify the characteristics that include the decay time, various source inputs that can create persistence, and whether or not amelioration techniques would help remove the unwanted signal.

\subsection{Decay Time}

Persistence is often quantified in terms of percentage of charge present at period of time after the source is removed. However, since persistence is actually a current source the percentage measurement is dependent on the integration time of the latency image. Thus all of our measurements are divided by the integration time and quoted in terms of current with a unit of electrons per second. Persistence can be thought of as "virtual dark current" and can be compared to a minimum amount of unwanted current, the lower limit being the amount of true dark current.

\begin{tabular}{|c|c|c|c|c|c|c|c|c|c|c|c|}
\hline \multicolumn{12}{|c|}{ Persistence Current Decay Data Set 1} \\
\hline Integration Time (sec) & 100 & 100 & 100 & 100 & 100 & 128 & 256 & 512 & 1024 & 1024 & 1024 \\
\hline Time from Illumination Removal (sec) & 54 & 270 & 486 & 702 & 918 & 2994 & 3258 & 3778 & 4810 & 6874 & 7906 \\
\hline Persistence Current (e-s / sec) & 19.680 & 2.480 & 1.360 & 0.920 & 0.640 & 0.250 & 0.250 & 0.211 & 0.160 & 0.121 & 0.113 \\
\hline \multicolumn{12}{|c|}{ Persistence Current Decay Data Set 2} \\
\hline Integration Time (sec) & 1 & 2 & 4 & 8 & 16 & 32 & 64 & 128 & 256 & 512 & 1024 \\
\hline Time from Illumination Removal (sec) & 6 & 16 & 28 & 44 & 68 & 108 & 180 & 316 & 580 & 1100 & 2132 \\
\hline Persistence Current (e-s / sec) & 92.000 & 38.000 & 19.000 & 13.000 & 9.500 & 6.375 & 3.938 & 2.500 & 1.406 & 0.773 & 0.398 \\
\hline
\end{tabular}

Table I. Decay Time Measurements for Persistence Current

We performed several experiments where the detector was exposed to high flux sources of various wavelengths and the amount of persistence signal was measured as function of time after the illuminating source was removed. The amount of trapped charge has an upper limit, Benson et al. (2000), so increasing the flux beyond a certain point does not increase the persistence effect. We also found that the wavelength of the incoming flux has no measurable effect on the persistence characteristics over the wavelength range tested, which was $1.1 \mu \mathrm{m}$ to $4.1 \mu \mathrm{m}$. This wavelength independent result is consistent with tests on other types of InSb arrays, Figer et al. (2004). The decay time measurements are summarized in Table I. The decay time data are plotted in Figure 2, which includes a fit to the data and the true dark current limit measured after the detector was maintained in a dark environment for greater than 72 hours.

\subsection{Source Flux and Fluence}

Persistence can result from saturating the array with too long of an exposure time or from high levels of incident flux on the array, independent of integration times. We compared the level persistence from both types of sources in order to know which of these are more important to avoid. Fluence is defined as flux multiplied by integration time. Figure 3 plots a comparison of the persistence current verses source intensity in units of full-well percentage. The saturation full well capacity of the Alladin-3 array is approximately $\sim 25,000 \mathrm{e}^{-\mathrm{s}}$. The data points plotted using the square symbols were a result of keeping the integration time constant while increasing the 
incident flux. The data plotted with the circle shaped symbols resulted from a relatively low incident flux while letting the detector saturate with increasing integration times. In both plots the persistence was measured immediately after the illumination was removed using an integration time of $10 \mathrm{sec}$. This comparison shows that incident flux is a more significant factor in generating latent charge than letting the array saturate from longer integrations. The data presented in figure 3 were acquired at $2.20 \mu \mathrm{m}$ (K-band) and data acquired at $1.25 \mu \mathrm{m}$ (J-band) was virtually the same. Longer wavelength versions of this test were not performed due to the difficulty of achieving a low incident flux environment at thermal infrared wavelengths

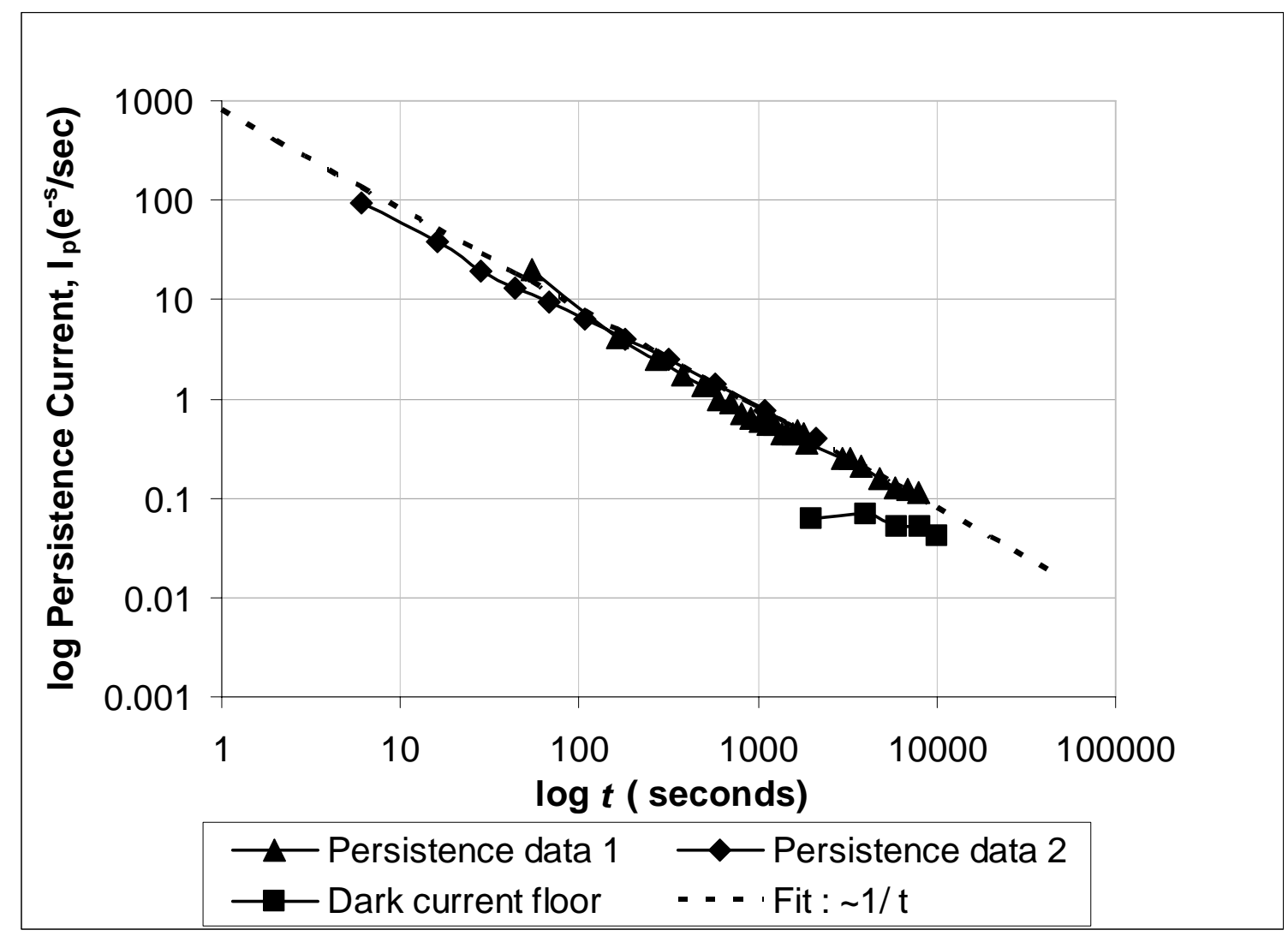

Figure 2. Persistence Current, Ip, Decay Rate

Charge persistence leaks into the measured signal of the detector at a rate that is proportional to the time after the illuminating source has been removed. The persistence current appears nearly as a straight line when plotted with $\log (t)$ vs $\log (I p)$. The decay rate of current is proportional to $1 / t$. The dark current limit is overlaid on this plot, which was measured after 72 hours of no light on the detector. These data indicate that a waiting period of greater than 10,000 seconds is required to allow the persistence to reach an acceptable level

\subsection{Removing the Trapped Charge with Continuous Resets}

There is anecdotal evidence that with certain types of arrays, the persistence can be removed or the decay time can be improved if the array is flushed, which is defined as series of reset-reads. We tested this technique by comparing decay times with and without flushing applied. After illuminating the array with the identical source, the persistence level was measured every hour but in one set of data, the array was reset and readout continuously in the period between samples. Figure 4 plots this comparison and shows that this type of attempt at ameliorating the 
charge not only failed but seemed to make things worse, presumably by raising the dark current level through internal heating of the array.

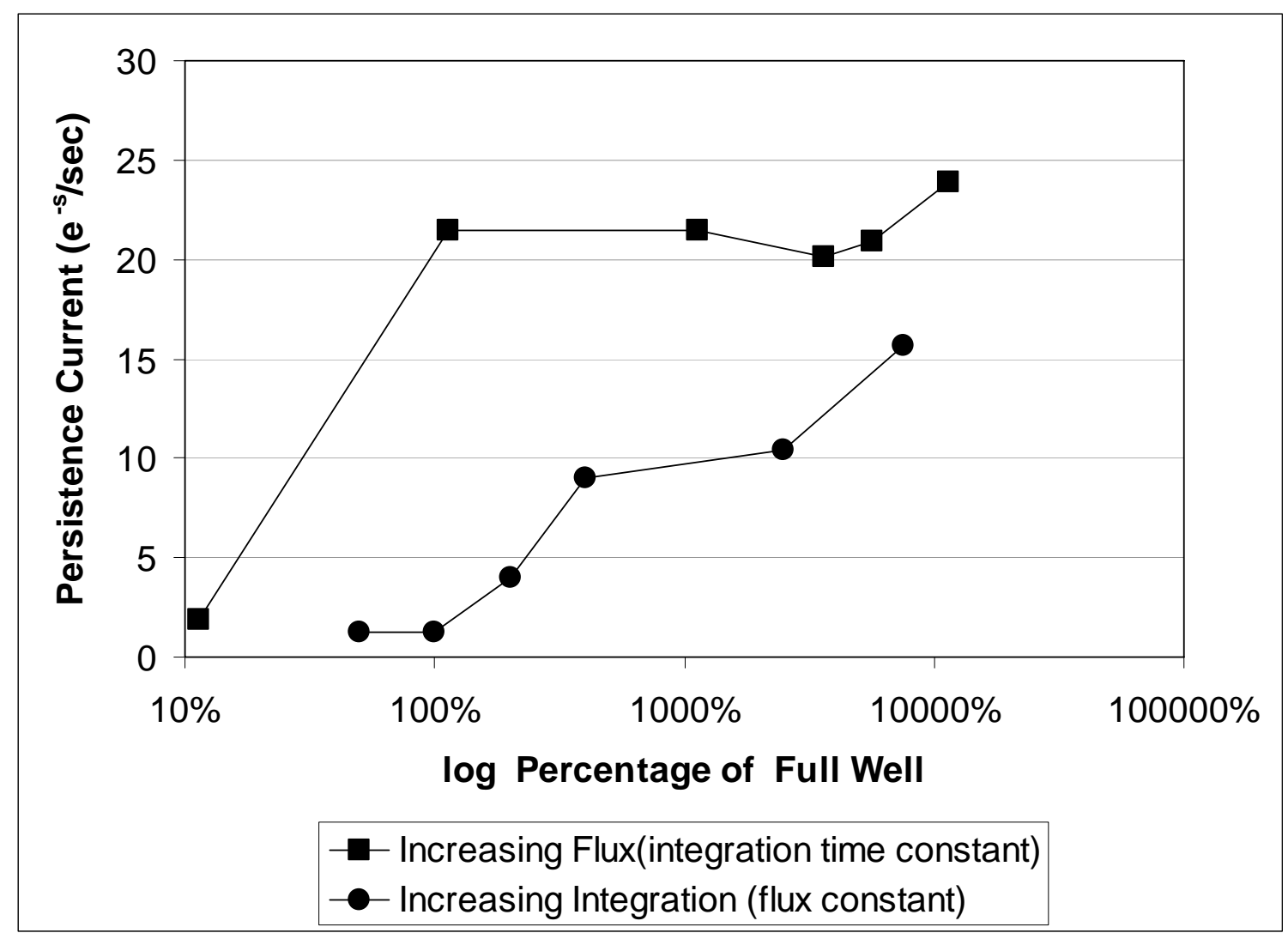

Figure 3. Comparison of Incident Flux and Fluence (flux x integration time) Effects on Persistence Current With integration time held constant $(1 \mathrm{sec})$ a light source was adjusted with varying ND filters to increase the flux incident on the array to beyond saturations levels (square symbols). Another test was performed by keep the flux source constant and increasing the integration times to beyond the saturation point (circular symbols). The residual current was measured in each case and plotted above. The plot shows that flux alone is a stronger source of persistence than fluence.

\section{Conclusions}

Persistence is a significant characteristic of detectors used for astronomy, particularly infrared hybrid arrays with InSb as the photo-sensitive material. Observing plans must account for this effect in order to keep the area where faint object scientific data will be gathered on the array protected from high flux sources. Saturation levels in this region of the array should also be avoided. Persistence can require up to 3 hours to return to acceptable levels of charge leakage near the normal dark current lower limit. Persistence is a current source and thus longer integrations are more susceptible to the negative consequences if its effects. The persistence current decay time is proportional to $t^{-1}$. High flux sources are more significant than high fluence sources and flushing the array with continuous reset-reads is not effective at ameliorating the trapped charge. 


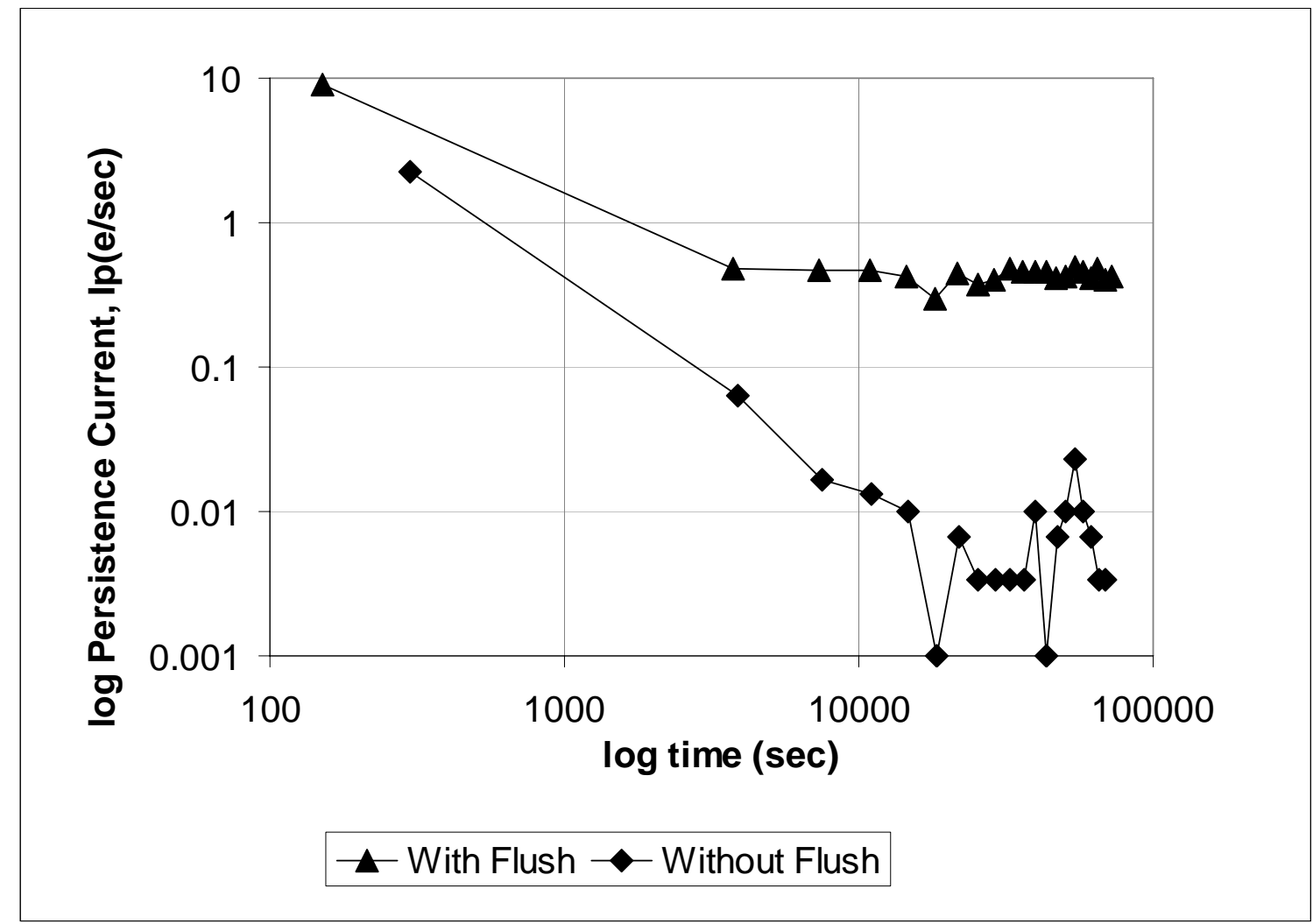

Figure 4. Effects of Flushing (Continuous Reset/Reads) on Persistence Decay

This plot not only indicates that continuous reset-reads fail to improve persistence decay, but actually makes things worse. Images were saved each hour with continues reads occurring in between the data sets in the one series and no reads or resets in the other. The decay rate did not have a significant difference between the two but the dark current floor apparently became worse when flushing. This may be due to local heating effects on the detector. The Aladin-3 was controlled to $29.0 \mathrm{~K}$ during these tests.

\section{References}

[1] Benson, Robert G.; Forrest, William J.; Pipher, Judith L.; Glaccum, William J.; Solomon, Steven L.: 2000, in Marija Strojnik; Bjorn F. Andresen; (Eds.), Infrared Spaceborne Remote Sensing VIII, Proc. SPIE Vol. 4131, p. 171-184.

[2] Figer, Donald F., et al.:2004, in Grycewicz, Thomas J.; McCreight, Craig R. (Eds.), Focal Plane Arrays for Space Telescopes ,Proc. SPIE, Volume 5167, pp. 270-301,..

[3] Fowler, Albert M.; Gatley, Ian; McIntyre, Paul; Vrba, Frederick J.; Hoffman, Alan W.:1996 in Randolph E. Longshore; Jan W. Baars; (Eds.), Infrared Detectors for Remote Sensing: Physics, Materials, and Devices Proc. SPIE Vol. 2816, p. 150-160.

[4] Solomon, Steven L.; Garnett, James D.; Chen, Hao: 1993, in Albert M. Fowler; (Ed), Infrared Detectors and Instrumentation, Proc. SPIE Vol. 1946, p. 33-45

[5] Solomon, Steven L.: 1999 "Charge Trapping in InSb Photodiode Arrays." Ph.D. Dissertation, University of Rochester, Rochester, N.Y. 